\title{
Locus-specific hypomethylation of the mouse IAP retrotransposon is associated with transcription factor-binding sites
}

\author{
Ken-ichi Shimosuga ${ }^{1,2}$, Kei Fukuda $^{1,3}$, Hiroyuki Sasaki $^{1}$ and Kenji Ichiyanagi ${ }^{1,4^{*}}$ (D)
}

\begin{abstract}
Background: Intracisternal A particle (IAP) is one of the most transpositionally active retrotransposons in the mouse genome, but its expression varies between cell types. This variation is believed to arise from differences in the epigenetic state (e.g., DNA methylation) of the $5^{\prime}$ long terminal repeat (LTR), where transcription starts. However, owing to the high copy number and high sequence similarity between copies, it is difficult to analyze the epigenetic states of individual IAP LTRs in a comprehensive manner.

Results: We have developed a method called Target Enrichment after Post-Bisulfite Adaptor Tagging (TEPBAT) to analyze the DNA methylation states of a large number of individual retrotransposon copies at once. Using this method, we determined the DNA methylation levels of $>8500$ copies of genomic IAP LTRs (almost all copies that we aimed to target by the PCR primers) in the sperm and tail. This revealed that the vast majority of the LTRs were heavily methylated in both sperm and tail; however, hypomethylated copies were more frequently found in the sperm than in the tail. Interestingly, most of these hypomethylated LTRs were solo-type, belonged to specific IAP subfamilies, and carried binding sites for transcription factors (TFs) that are active in male germ cells.

Conclusions: The current study revealed subfamily- and locus-specific hypomethylation of IAP LTRs, and suggests that binding of TFs is involved in the protection from DNA methylation, whereas the IAP internal sequence enhances methylation. Furthermore, the study demonstrated that TEPBAT offers a cost-effective method for a variety of DNA methylome studies that focus on retrotransposon sequences.
\end{abstract}

Keywords: DNA methylome, Intracisternal A particle, Endogenouse retrovirus, Transcription factor, Spermatogenesis, Mouse

\section{Background}

Approximately $40 \%$ of the mammalian genome comprises several million copies of retrotransposons [1], which include long terminal repeat (LTR) retrotransposons, long interspersed elements, and short interspersed elements. These retrotransposons are amplified by retrotransposition, a process in which their transcribed RNA is utilized to make a DNA copy by reverse transcription. It has been reported that retrotransposition causes heritable diseases, such as hemophilia A and B, muscular

\footnotetext{
*Correspondence: ichiyana@agr.nagoya-u.ac.jp

'Division of Epigenomics and Development, Medical Institute of Bioregulation, and Epigenome Network Research Center, Kyushu University, 3-1-1 Maidashi, Higashi-ku, Fukuoka 812-8582, Japan

${ }^{4}$ Laboratory of Genome and Epigenome Dynamics, Department of Applied Molecular Biosciences, Graduate School of Bioagricultural Sciences, Nagoya University, Nagoya 464-8601, Japan

Full list of author information is available at the end of the article
}

dystrophy, and $\mathrm{X}$-linked agammaglobulinemia in humans [2]. In mice, $10-12 \%$ of spontaneous mutant alleles arise as a result of the retrotransposition of LTR retrotransposons, such as intracisternal A particle (IAP), early transposon (ETn), and MusD [3]. To diminish the transpositional activity, retrotransposon expression can be epigenetically regulated at the transcriptional level by DNA methylation at $\mathrm{CpG}$ sites and by repressive histone modifications. A knockout mutation of the mouse Dnmt1 gene, which encodes a maintenance-type DNA methyltransferase, has been shown to cause derepression of IAP in whole embryos because of a passive loss of DNA methylation [4]. Likewise, several LTR retrotransposons are derepressed in mouse embryonic stem cells having deletion of the Setdb1 gene, which encodes a protein methyltransferase acting on the lysine- 9 residue of histone H3 
(H3K9). The derepression is attributable to the loss of H3K9 trimethylation at the LTRs $[5,6]$.

The mouse genome contains approximately 4000 copies of full-length or nearly full-length IAP, which consist of two LTRs at both ends and an internal sequence carrying gag, pro, and pol genes. In addition, the genome contains approximately 5000 copies of solo LTRs of IAP, where an LTR alone is present. The sequence of IAP LTR is 300- to 450-bp long, and contains $15-25$ CpG sites. In somatic cells, the vast majority of these $\mathrm{CpG}$ sites are heavily methylated, and consequently, IAP expression level is very low [4]. However, it has been reported that a small fraction of LTRs escape methylation with significant variation between individuals; sometimes they behave as metastable epialleles (i.e., LTR copies in the $A^{v y}, A^{i a p}$, and $A x$ in $^{f u}$ loci) $[7,8]$. Conversely, higher IAP expression is detected in preimplantation embryos (from the 8-cell to blastocyst stages) [9] and in prospermatogonia and spermatogonia [10], which are precursors of spermatozoa. Although DNA methylation levels in IAP copies are indeed low in blastocysts, those in spermatogonia are high [11-13]. Therefore, it is possible that in spermatogonia, some specific IAP copies are hypomethylated to serve as a source of IAP expression, whereas the vast majority remains highly methylated. It would be therefore interesting to analyze the methylation levels in individual IAP copies in a genome-wide manner, rather than to analyze them in bulk (via PCR using primers for the IAP consensus sequence). The development of the whole-genome bisulfite shotgun sequencing method offers an opportunity to analyze DNA methylation levels in individual IAP copies (i.e., the IAP methylome). However, the cost to obtain sufficient sequence depth is high. To reduce the cost while maintaining the comprehensive depth that is required, a method called high-throughput targeted repeat element bisulfite sequencing (HT-TREBS) [14] was recently developed. The study confirmed the high level of DNA methylation at vast majority of IAP LTRs, while a small subset are hypomethylated. However, the mechanism underlying their hypomethylation remains unknown.

In the present study, we determined the IAP methylomes in germ and somatic cells using a method where IAPs and their flanking sequences were selectively amplified using a random primer and an IAP-specific primer after bisulfite treatment of genomic DNA. Deep sequencing analysis revealed that specific IAP subfamilies were hypomethylated, whereas a vast majority were highly methylated. Most of the hypomethylated copies were solo LTRs and carried binding motifs for specific transcription factors (TFs). We discuss a possible role of TFs in protecting these copies from methylation and a role for the internal sequence in recruiting methylation enzymes.

\section{Results}

\section{Determination of DNA methylation levels of IAP LTR} copies by the TEPBAT method

To selectively obtain bisulfite sequencing data for the IAP LTR sequences, we developed a method that was modified from the PBAT library preparation method designed for whole-genome bisulfite sequencing [15]. In our method (Fig. 1a), designated as TEPBAT (Target Enrichment after Post-Bisulfite Adaptor Tagging), the first DNA strand was synthesized using bisulfite-treated DNA as a template and the tag-plus-random primer, which consisted of a random tetramer and a specific tag sequence. The random tetramer end of the primer enabled genome-wide synthesis of the first DNA strand. In the second step, IAP-containing regions were selectively amplified by PCR with the IAP-specific primer and tagsequence primer (which was almost same as the tagplus-random primer, but did not contain the random tetramer sequence).

Genomic copies of the IAPLTR1_Mm_LTR (referred to as IAPLTR1), IAPLTR1a_MM (IAPLTR1a), IAPLTR2_Mm (IAPLTR2), IAPLTR2a2_Mm (IAPLTR2a2), and IAPL TR2b subfamilies comprise more than half of the total IAP LTRs in the genome (see Fig. 2a) and are less diverged because of recent retrotransposition. Therefore, in the present study, we targeted these copies for methylation analysis. To selectively amplify these copies, the IAP-BS1 and IAP-BS2 primers were designed in regions that are highly conserved among the subfamilies (Fig. 1b). IAPBS1 was designed in the reverse orientation to amplify the $5^{\prime}$ region and the upstream flanking sequence, whereas IAP-BS2 was designed in the forward orientation to amplify the 3' region and the downstream flanking sequence. We note that the IAP-BS2 primer could hybridize to IAPEY_LTR (IAPEY) and IAPEY2_LTR (IAPEY2) weakly. The PCR products were ligated to the sequencing adaptor, and paired-end deep sequencing was performed on HiSeq2500 so that one of the paired reads facilitated mapping uniquely to the genome, whereas the other contained the IAP sequence.

To investigate and compare the IAP methylation profiles of germ and somatic cells, we prepared genomic DNAs from the sperm and tail of the same male mouse (the C57BL6/J strain). Using these DNAs, we obtained 86 and 81 million sequencing read pairs, respectively. After removing the primer sequences (including the region of the random tetramer) and low-quality nucleotides, the read pairs were mapped to the mouse genome sequence to call the methylation state of each CpG site (see Methods). On average, about $10 \mathrm{CpG}$ sites in an LTR were covered by the reads with an average sequencing depth of about 320 (yielding about $320 \times 10=3200$ methylation calls). To calculate the methylation level at individual IAP LTRs (expressed as a fractional value 
a IAPLTR $1 \mathrm{Mm}$ LTR IAPLTR $1 \bar{a}$ MM $\bar{M}$ IAPLTR $2 \mathrm{~b}$ IAPLTR2a2_Mm IAPLTR2 Mm

IAPLTR1_Mm_LTR IAPLTR $1 \bar{a}+M \bar{M}$ IAPLTR $2 b$ IAPLTR2 $22 \mathrm{Mm}$ IAPLTR2_Mm

IAPLTR 1 Mm LTR IAPLTR1ā_MM IAPLTR $2 \mathrm{~b}$ IAPLTR2 $22 \mathrm{Mm}$ IAPLTR2 $\mathrm{Mm}$

IAPLTR1_Mm_LTR IAPLTR 1a MM IAPLTR $2 b$ IAPLTR2 $22 \mathrm{Mm}$ IAPLTR2_Mm

IAPLTR 1 Mm LTR IAPLTR1 $1 \bar{a}-M \bar{M}$ IAPLTR $2 b$ IAPLTR2 $22 \mathrm{Mm}$ IAPLTR2_Mm

IAPLTR1_Mm_LTR IAPLTR1a MM IAPLTR $2 b$ IAPLTR 2 a $2 \mathrm{Mm}$ IAPLTR2_Mm
TGTTGGGAGCCGCGCCCACATTCGCCGTTACAAGATGGCGCTGACAGC-TGTGTTCTAAGTGGTAAACAAATAATCTGCGCATGTG TGTTGGGAGCCGCGCCCACATTCGCCGTTACAAGATGGCGCTGACAGC-TGTGTTCTAAGTGGTAAACAAATAATCTGCGCATGTG TGTGGGGAGCCGCCCTCACATTCGCCGTTACAAGATGGCGCTGACATCCTGTGTTCTAAGTGGTAAACAAATAATCTGCGCATGTG TGTGGGGAGCCGCCCTCACATTTGCCATTATAAGATGGCGCTGACAGC-TGTGTTCTAAGTGGTAAACA--TAATCTGCACACGTG TGTGGGGAGCCGCCCTCACATTCGCCGTTGCAAGATGGCGCTGACATCCTGTGTTCTAAGTGGTAAACAAATAATCTGCGCATGTG

IAP-BS1

CCAAGGGTATCTTATGACTACTTGTGCTCTGCCTTCCCCGTGACGTCAACTCGGCCGATGGGCTGCAGCCAATCAGGGAGTGACAC CCGAGGGTGGTTCTCCACTCCATGTGCTCTGCCTTCCCCGTGACGTCAACTCGGCCGATGGGCTGCAGCCAATCAGGGAGTGACAC CCAAGGGTAGTTCTCCACTCCATGTGCTCTGCCTTCCCCGTGACGACAACTCGGCCGATGGGCTGCAGCCAATCAGGGAGCGACAC C-AGGGGCAGTTTTCC-CGCCATGTGTTCTGCCTTTCTCGTGATGACAACTGGGCCGATGGGCTGCAGCCAATCAGGGAGTAATAC CCAAGGGTAGTTCTCCACCCCATGTGCTCTGCCTTCCCCGTGACGACAACTCGGCCGATGGGCTGCAGCCAATCAGGGAGTGATAC

GTCCGAGGCGAAGGAGAATGCTCCTTAAGAGGGACGGGGTTTCGTTTTC-TCTCTCTCTTGCT-TCTTGCTCTCTTGCTT-CTTGC GTCCTAGGCGAAGGATAATTCTCCTTAATAGGGACGGGGTTTCGTTTTC-TCTCTCTCTTGCT-TCTT-GTCCTAGGCGGAGGATAATTCTCCTTAAAAGGGACGGGGTTTCGCCAT--TCTCTCTCTTGCT-TCTT---------------GTCCTAGGCGGAGGATAATTCTCCTTAAAAGGGACGGGGTTTTGCCATTTTCTCTCTCTTGCTTTCTTGTTCTCTCTCTTGCTTTC GTCCTAGGCGGAGGATAATTCTCCTTAAAAGGGACGGGGTTTCGCCATT--CTCTCTCTTGCTCTCTTGCGCTCTTGCTCTCTTGC

TСТС

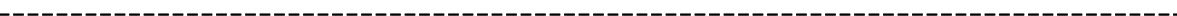

T-TGTTTTCTCGTTCTTGCTCTCTT-------_GCTTTCTTGTTCTTGTTCTTT-TTCTTGCTTTCTTGCTCTCTCTTGCTCTCTT TCTGCTCTTGCGCTCTGGCTCCTAAAGATGTAAGCAATAGAGCTCTTGCTCTGCGCTCTTGCGCTCTTGCGCTCTTGCGCTCTGGC

IAP-BS2

--------------------------"TTGCTTCTTGCACTCTGGCTCCTGAAGATGTAAGCAATAAAGCTIT-GCCGCAGAAGAT

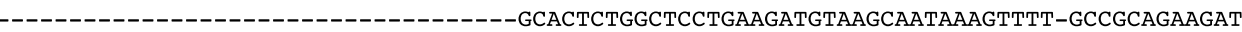

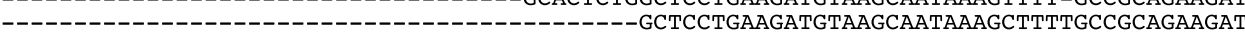
GCT----_---_---_---CTCTTGCTCTCTTGCTCTCTGGCTCCTGAAGATGTAAGCAATAAAGCTTTTGCCGCAGAAGAT TCCTAAAGATGTAAGCAATAGAGCTCTTGCTCTCTTGCTCTCTGGCTCCTGAAGATGTAAGCAATAAAGCTTT-GCCGCAGAAGAT

TCTGGTCTGTGGTGTTCTT-CCTGGCCGGTCGTGAGAACGCGTCTAATAACA TCTGGTCTGTGGTGTTCTT-CCTGGCCGGGCGTGAGAACGCGTCTAATAACA TCCGGTTTGTTGCGTTCTT-CCTGGCCGGTCGCGAGAACGCGTGTAAGA--TCCGGTTTGTTGCGTTCTTTCCTGGCCGGTCGCGAGAACGCGTGTAAGA--TCCGGTTTGTTGCGTTCTT-CCTGGCCGGTCGCGAGAACGCGTGTAAGA---

b

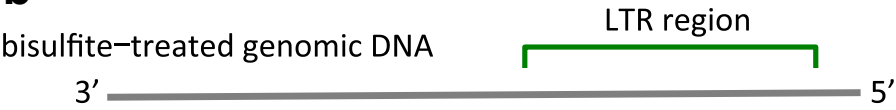

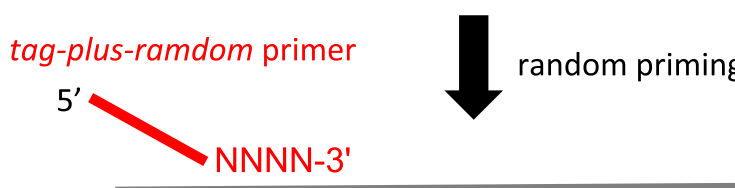
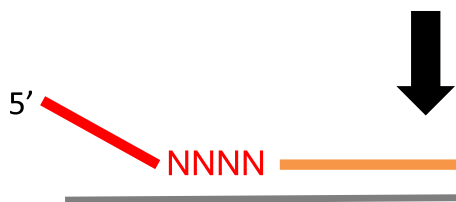

1st strand syntesis $5^{\prime}$

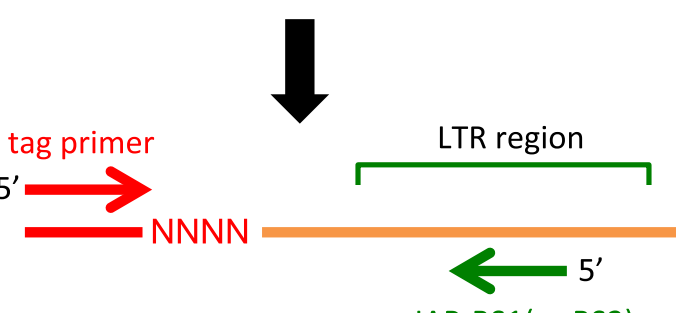

IAP-BS1(or BS2)

PCR

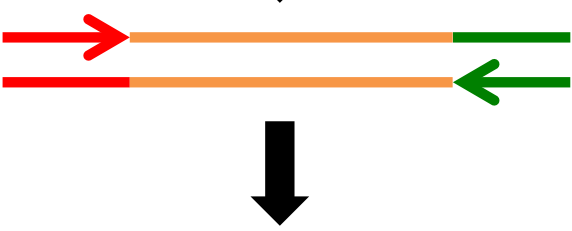

Library construction

for deep sequencing

Fig. 1 Experimental design of TEPBAT. a Nucleotide sequence alignment of the consensus sequences of IAPLTR1, IAPLTR1a, IAPLTR2, IAPLTR2a2, and IAPLTR2b. The primer regions and orientations (IAP-BS1 and IAP-BS2) used in TEPBAT are indicated as green arrows. b Experimental design to enrich IAP regions in the bisulfite-treated genomic DNA. After bisulfite treatment, the first DNA strand was synthesized using the tag-plus-random primer, and the IAP regions were amplified by PCR using the specific primer (green arrow) and tag primer (red arrow) 


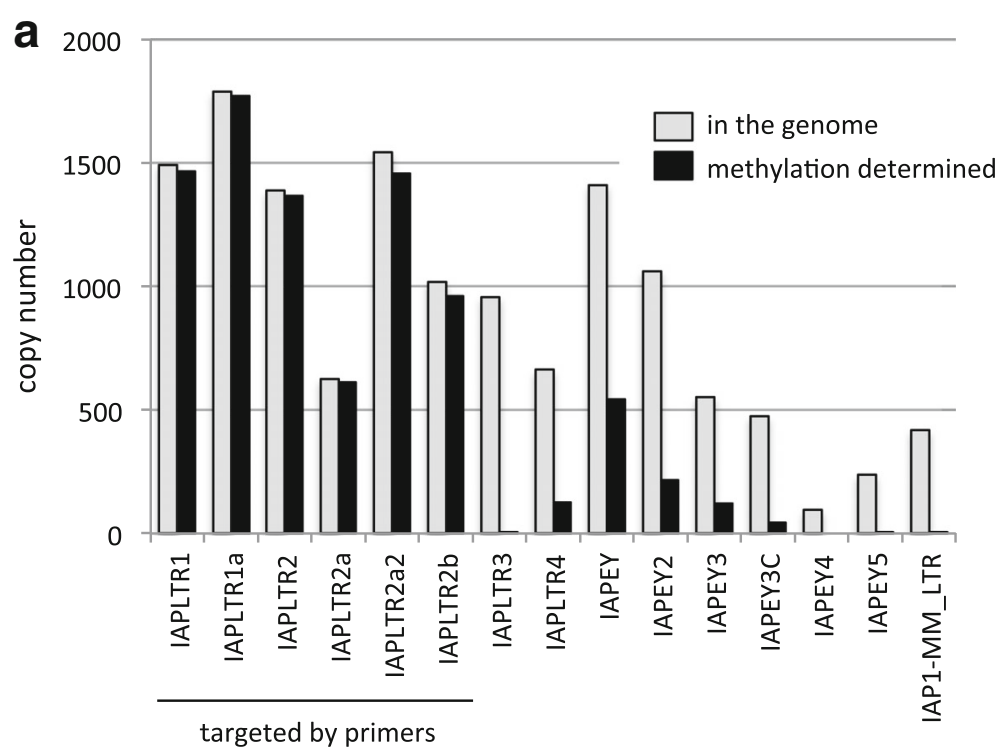

b chr7:138688945-138689342

TEPBAT: 0.93 BS-PCR: 0.99

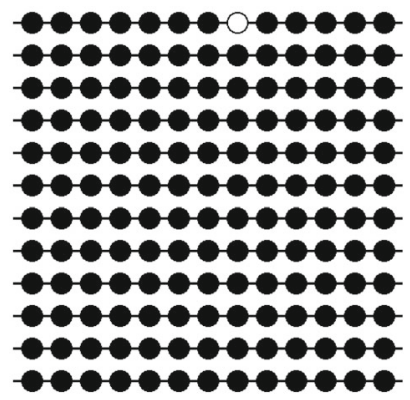

d

chr9:83949452-83949868

TEPBAT: 0.93

BS-PCR: 0.94

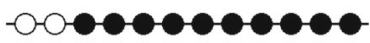
$-0-00000000$ $000000000-$ - $-0000-000-$ - $-000-000-00$ -000-00-00-0000000000 - 100000000 0000000000 $-000-000-00$ - 00000000 $-000-000-00$ $-000000000$
C chr1:19629200-19629572

TEPBAT: 0.89 BS-PCR: 0.86

-OO-OO-OO$-100-100-0$ - $1000-000-0$ $-000-000$

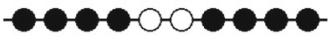
$-100000-100$ $-00000000$ $-0000000$ $-1000000$ $-00000000$ $-1000000$ $-1000000$ $-10000$

e

chr1:87466910-87467259

TEPBAT: 0.53

BS-PCR: 0.55

-OC-O-O-O-O-O - O-O-00-00-00-

o-0-0-0-00-0-

$-0-0-0-000-0$

-O-O-O-O-O-O-

$0-000-0000$

-O-O-0000-00

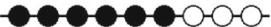

$-00000-000$

- $-0000-00$

$-000-00$

- $-000-00-$

$-0-000-00$

$-1000-00$

$-00000$

$-000-00$

$-000000$

Fig. 2 (See legend on next page.) 
(See figure on previous page.)

Fig. 2 DNA methylation levels of individual IAP LTR copies. a The number of LTR copies of the subfamilies. The numbers of the total genomic copies are shown in gray and those for which methylation levels were analyzed (total CpG methylation calls of $\geqq 20$ ) are shown in black. b-e Bisulfite-PCR results for selected loci in the tail DNA. Closed and open circles indicate methylated and unmethylated CpG sites, respectively. Each row represents a clone of bisulfite-PCR products. The genomic location $(\mathrm{mm} 10)$ of the loci and their methylation levels (expressed as a fractional value) determined by TEPBAT and bisulfite-PCR (BS-PCR) are shown

[0.00-1.00] and calculated by dividing the methylated cytosine calls by a sum of methylated and unmethylated cytosine calls), the methylation levels at $\mathrm{CpG}$ sites within an LTR were averaged. LTRs with $<20 \mathrm{CpG}$ methylation calls were excluded from the analysis, retaining the methylation data of 8698 and 8517 LTRs for the sperm and tail, respectively. For comparative analysis, we focused on 8153 LTRs whose methylation levels could be analyzed in both samples. These LTRs included $>97 \%$ of genomic copies of the targeted IAP subfamilies (IAPLTR $1 / 1 \mathrm{a} / 2 / 2 \mathrm{a} / 2 \mathrm{a} 2 / 2 \mathrm{~b}$ ), and 40 and $20 \%$ of genomic IAPEY and IAPEY2 LTRs, respectively (Fig. 2a). To validate the acquired methylation data, we determined the methylation levels of selected LTR loci by bisulfite-PCR using the tail DNA. This data was consistent with the TEPBAT data (Fig. 2b-e).

High-throughput analysis revealed that 6677 (80\% of the total LTRs analyzed) and 7726 (93\%) LTRs exhibited methylation levels of $>0.8$ in the sperm and tail, respectively (Fig. 3a, b). This is consistent with the notion that IAP LTRs are generally methylated. However, a small fraction of LTRs showed medium $(0.2-0.8)$ and low $(\leqq 0.2)$ methylation levels. Hypomethylation (methylation level $\leqq 0.2$ ) was tissue-specific, and only three LTR copies were hypomethylated in both the sperm and tail (Fig. 3c). In the sperm, 43 and 1612 LTR copies showed low and medium methylation levels, respectively (Fig. 3a), whereas 14 and 612 copies showed low and medium methylation levels in the tail, respectively (Fig. 3b). Therefore, although generally hypermethylated, the IAP LTR methylation level is relatively low in germ cells as compared to somatic cells.

The IAP LTR methylation levels could vary between individuals. Therefore, different individuals were analyzed for several LTR loci in the tail and sperm by bisulfite-PCR (Fig. 3d). The methylation levels at locus3 (IAPLTR2) in tail showed significant variation between individuals ( 0.47 in individual \#2, whereas 0.03 in individual \#1 and $<0.01$ in individuals \#3 and \#4). However, the methylation levels at other loci were largely conserved among the four individuals analyzed.

Solo LTRs of specific subfamilies display hypomethylation We investigated whether each subfamily showed specific methylation profiles. The median methylation level was $>0.8$ for all subfamilies (Fig. 4a, b); however, we found that IAPEY and IAPLTR2a2 in the sperm and IAPLTR2 in the tail contained some hypomethylated loci. Indeed, out of the 43 hypomethylated LTRs in the sperm (methylation level of $\leqq 0.2), 23$ (53\%), and 13 (30\%) belonged to IAPEY and IAPLTR2a2, respectively (Fig. 4c). In the tail, 7 (50\%) out of the 14 hypomethylated LTRs belonged to IAPLTR2 (Fig. 4d). Therefore, even though most subfamilies showed high methylation levels, a fraction of copies of the specific subfamilies were hypomethylated. IAPEY is a relatively young subfamily accumulated in the Y chromosome [16] with $60 \%$ of $\sim 1400$ genomic copies being in the $\mathrm{Y}$ chromosome (Additional file 1: Figure S1). Because the fraction of analyzed IAPEY copies was small (Fig. 2a), it should be noted that the number of hypomethylated IAPEY copies may be underestimated. The Y-chromosome copies are more divergent than those in autosomes (Additional file 1: Figure S1), and most (75\%) of the copies of which methylation levels were determined reside in autosomes. Consequently, almost all of the copies showing hypomethylation in sperm are also autosomal.

Even in these subfamilies, most loci were heavily methylated. To elucidate the mechanism of hypomethylation, we analyzed the sequence features of the hypomethylated loci. First, because LTR sequences exist as a terminal repeat or as a solo LTR, we determined whether these features affect methylation levels. We manually annotated all IAP LTRs in the genome as 5' LTR, 3' LTR, or solo LTR (Additional file 2: Table S1). The 8153 LTRs, for which methylation levels were determined in both tissues, included comparable numbers of 5' LTRs, 3' LTRs, and solo LTRs (2820, 2760, and 2573 copies, respectively). However, solo LTRs were significantly enriched with hypomethylated loci; for example, $36(84 \%)$ and $10(71 \%)$ of hypomethylated loci in the sperm and tail, respectively, were solo LTRs (Fig. 5a, b). On the other hand, $5^{\prime}$ and $3^{\prime}$ LTRs were less frequently hypomethylated. For these hypomethylated copies, it was generally observed in both sperm and tail that only one LTR was hypomethylated whereas the other in the same element was hypermethylated (Fig. 5c, d), suggesting that methylation levels of the $5^{\prime}$ and $3^{\prime}$ LTRs in an element are regulated independently of each other.

It should be noted that even for solo LTRs of the specific subfamily, not all loci were hypomethylated. Therefore, for these solo LTRs, we investigated whether some genomic and epigenomic features were associated with hypomethylation. However, we did not find a strong 


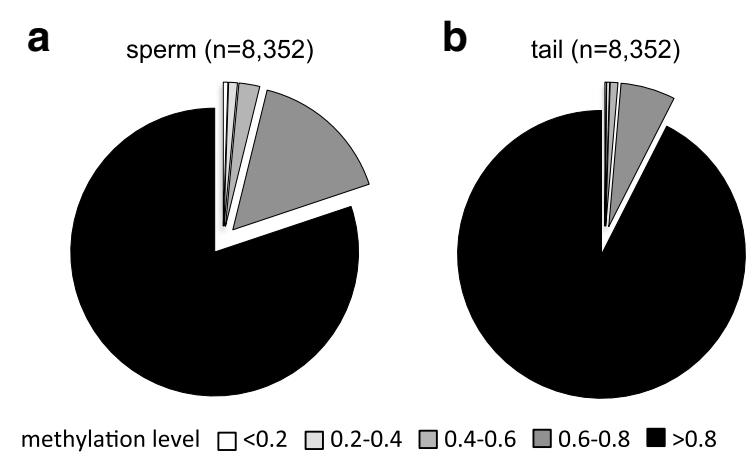

C

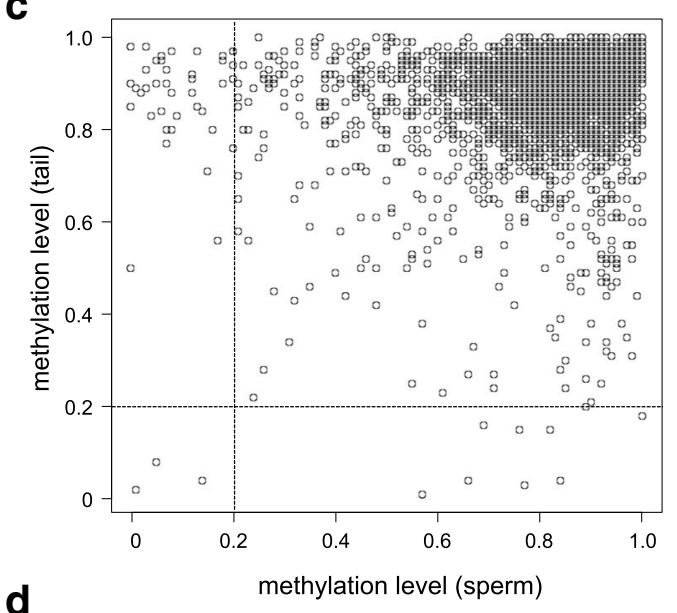

d

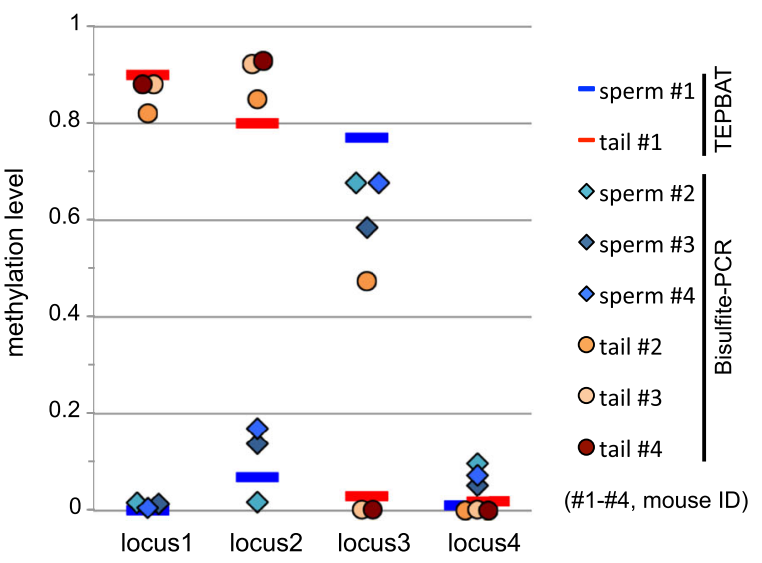

Fig. 3 IAP LTR methylation landscape. a, b Pie charts of LTR copies categorized by their methylation levels in the sperm (a) and in the tail (b). Methylation levels are indicated at the bottom. c Scatter plot of DNA methylation levels of IAP LTR copies $(n=8153)$ in the sperm and tail ( $x$ - and $y$-axis, respectively). $\mathbf{d}$ Bisulfite-PCR results for selected loci in the sperm and tail in different individuals (mouse ID \#2, \#3, and \#4). These individuals are different from the one (mouse ID \#1) used for TEPBAT analysis. Brue (sperm) and red (red) bars indicate the methylation levels determined by TEPBAT. Blue (sperm) and red (tail) circles indicate the methylation levels of three individuals determined by sequencing of 10 to 16 PCR clones. The genomic location $(\mathrm{mm} 10)$ of the loci are as follows: locus1, chr8:42,217,148-42,217,495 (IAPEY); locus2, chr18:87,502,570-87,503,025 (IAPLTR2a2); locus3, chr13:4,942,6524,943,122 (IAPLTR2); locus4, chr3:96,489,247-96,489,584 (IAPLTR1) correlation while analyzing their locations relative to genes (i.e., promoter, exon, intron, and intergenic), the distance to the nearest transcriptional start site, GC content of their neighboring regions (in 1-, 10-, or 100-kb bins), the number of $\mathrm{CpG}$ sites in their neighboring regions (in 1-, 10-, or 100-kb bins), the nucleotide divergence compared to the respective consensus sequences, methylation levels of their flanking regions in sperm [17], or their methylation levels in primordial germ cells at embryonic day 13.5 [18] (data not shown).

\section{Hypomethylated loci have specific sequences}

The sequences of LTR copies even of the same subfamily display slight variations. We analyzed the LTR sequences to see whether specific sequences are associated with hypomethylation. We aligned the sequences of the 11 IAPLTR2a2 sequences determined to be hypomethylated in the sperm, with randomly selected 34 hypermethylated (methylation levels of about 0.92) IAPLTR2a2 sequences. Clustering of the sequences by the neighborjoining method using Mega5 [19] revealed that these 45 sequences were divided into two major clades (Fig. 6a), one of which contained most of the hypomethylated LTRs. These were designated as the hypomethylated clade. Likewise, the 20 hypomethylated (in the sperm) and 22 randomly selected hypermethylated IAPEY sequences were divided into two clades, where the hypomethylated sequences were clustered together (Fig. 6b). The five hypomethylated (in the tail) and 20 randomly selected hypermethylated IAPLTR2 sequences were divided into two clades; the hypomethylated sequences were again clustered together (Fig. 6c).

In each case, the clear clustering (bootstrap values $>86$ ) suggested the presence of single nucleotide variations (SNVs) or sequence blocks that discriminate the two clades. Indeed, we identified several nucleotide positions that were conserved in the hypomethylated clade but not in the other clade. Given the possibility that sequence differences may result in differences in binding of TFs, we searched sequence motifs for TF binding by FIMO [20]. This revealed that eight TF-binding motifs (Prop1, Spi1, Ubp1, Hnf4g, Mitf, Maz, Mafk, and Nf212) were significantly enriched in the hypomethylated clade of IAPLTR2a2. In particular, the motifs for Maz and Ubp1 were absent in all (Maz) or most (Ubp1) of hypermethylated sequences, whereas all hypomethylated sequences carried multiple motif sequences. These hypomethylated clade-specific Ubp1 and Maz-binding motifs were all located in the R region of the LTR (Fig. 7), which is known to play a role in transcriptional regulation and shows extensive sequence variation [21]. It is noteworthy that out of the eight TFs mentioned above, Maz and Ubp1 are expressed in spermatogenic cells in the published transcriptome data [22]. Therefore, it is 


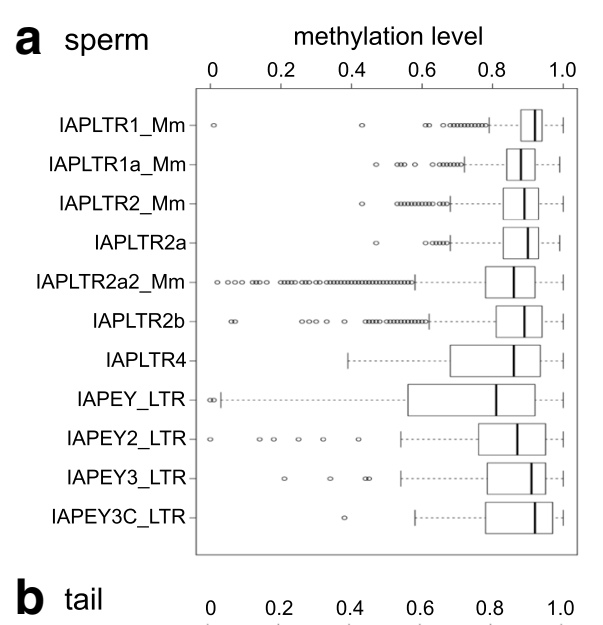

C methylation level of $<0.2($ sperm, $n=43)$
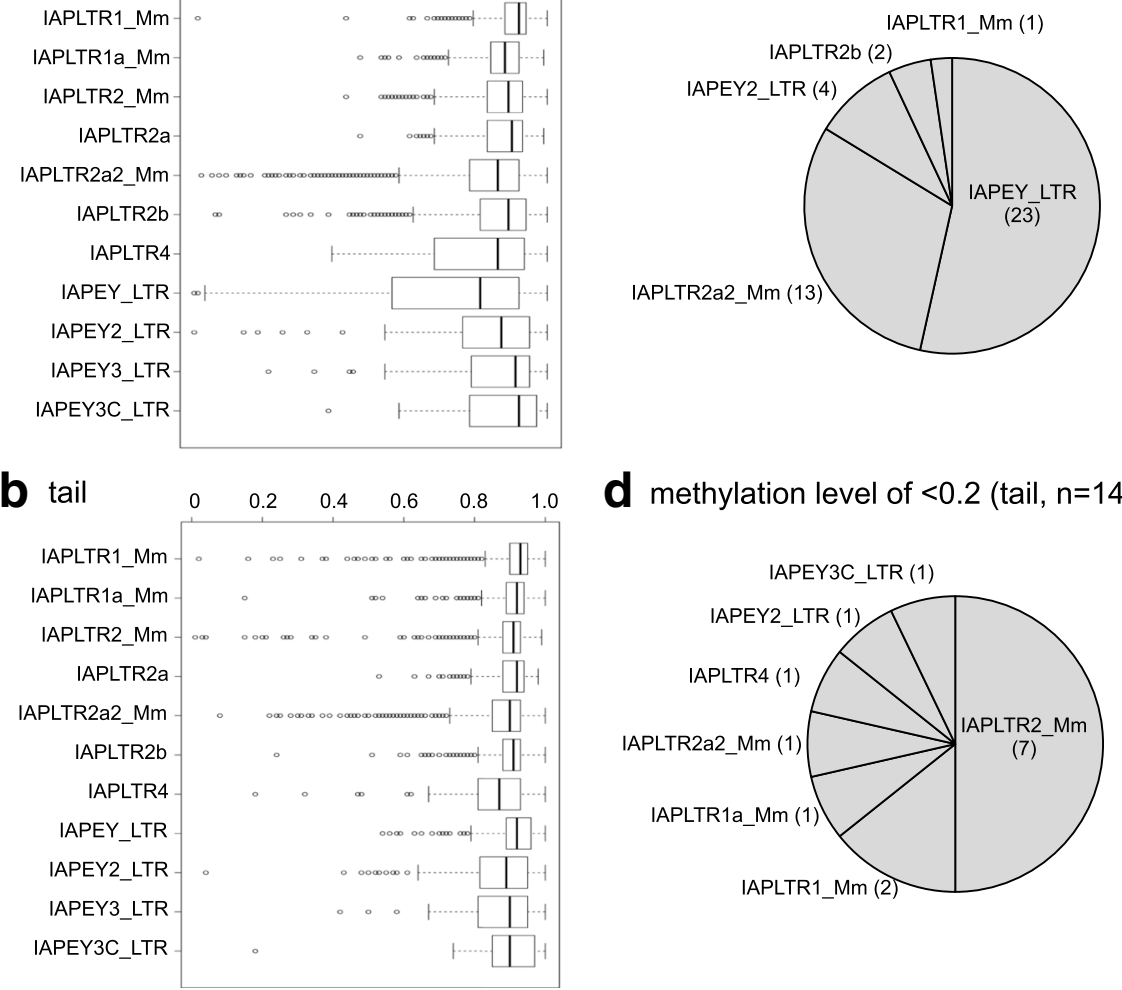

d methylation level of $<0.2$ (tail, $n=14)$

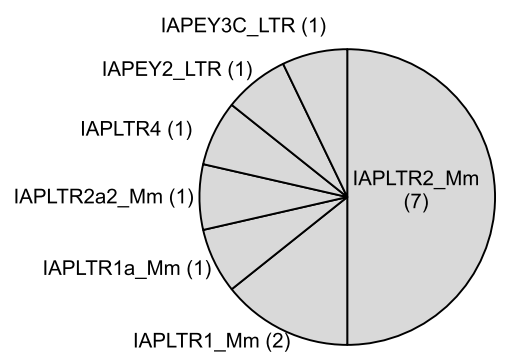

Fig. 4 Specific subfamilies are enriched in hypomethylated copies. $\mathbf{a}$, b Box plots of DNA methylation levels of IAP subfamilies in the sperm (a) and in the tail (b). c, d Subfamily distributions of hypomethylated copies (methylation level $<0.2)$ in the sperm (c) and in the tail (d) are shown in pie charts. The numbers in parentheses indicate actual copy numbers

conceivable that the binding of these TFs during spermatogenesis reduces the methylation level of their binding sites in these cells and spermatozoa. Interestingly, the hypomethylated sequences tend to have more CpG sites than hypermethylated sites, especially around the TF binding motifs (Fig. 7).

For the IAPEY subfamily, we also identified cladespecific SNVs, but could not identify clade-specific TFbinding motifs. In contrast, for the IAPLTR2 subfamily, we identified Plag1 and Sp1 binding motifs that were specific to the hypomethylated clade. The expression of these TFs in the adult tail is unknown.

\section{Discussion}

In the present study, we developed a method called TEPBAT to effectively analyze DNA methylation levels of genomic copies of a specific retrotransposon in bulk. In this method, a single lane of a HiSeq run was sufficient to obtain methylation data of thousands of genomic IAP LTR copies. It seems conceivable that other interspersed repeats can also be analyzed by TEPBAT if appropriately designed primers are used.
Although most of the genomic IAP LTR copies were heavily methylated in the sperm and tail, we found that LTRs of a specific subfamily exhibited a tendency to be hypomethylated, especially when specific sequences were present. This implies that the epigenetic state of IAP is dictated by the genetic sequence. Indeed, we found that several TF-binding motifs are specifically associated with hypomethylated sequences, which suggests that TF binding interferes with DNA methyltransferases, and subsequent methylation modifications (Fig. 8). A similar association between variation of TF-binding motifs and methylation levels has been observed for unique sequences as well [23-26]. However, not all copies in the hypomethylated clade were hypomethylated, suggesting that additional factor(s) may also be involved in the regulation of IAP methylation.

In contrast to solo LTRs, hypomethylation of $5^{\prime}$ and 3' LTRs was very infrequent. A possible explanation would be that the internal sequence of IAP ( $\mathrm{gag}$, pro, pol, and $5^{\prime}$ and $3^{\prime}$ untranslated regions) carry a sequence(s) bound to a protein(s) important for de novo and/or maintenance of DNA methylation. This type of methylation regulation may include Krüppel-associated 


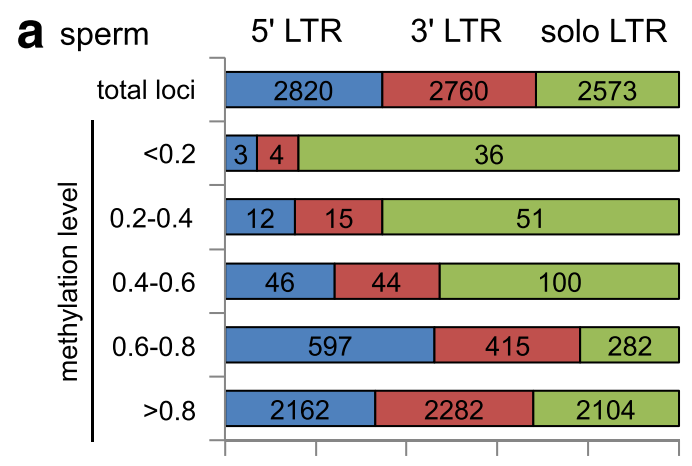

\section{LTR pair comparison (sperm)}
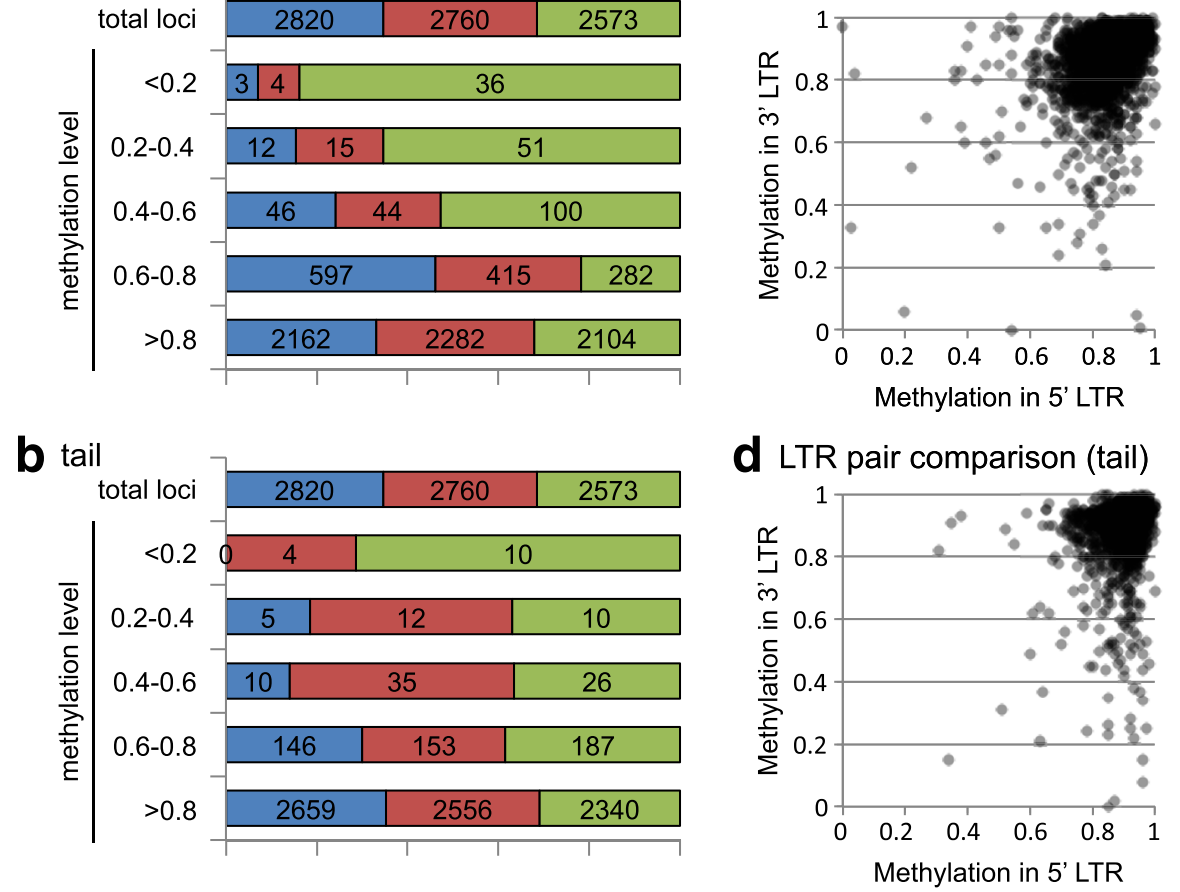

Fig. 5 LTR features and DNA methylation. The LTR copies ( $n=8153$ ) are categorized into 5' LTR (blue), 3' LTR (red) and solo LTR (green) and into five groups according to their methylation levels in the sperm (a) and in the tail (b). The numbers indicate actual copy numbers. (c, $\mathbf{d})$ Comparison of methylation levels in sperm (c) and tail (d) in the two LTRs within the same elements. For IAP copies having both 5' and 3' LTRs, the methylation levels in the 5' LTRs ( $x$ axis) are plotted against those in the 3' LTRs (y axis)

box containing zinc finger proteins (KRAB-ZFPs) that regulate the epigenetic state of their binding sequences. KRAB-ZFPs can bind DNA in a sequence-specific manner via the ZF domain, while they can also bind the KAP1 protein via the KRAB domain [27, 28]. The KAP1 protein in turn binds SetDB1, histone deacetylases, and DNA methyltransferases; therefore, the KRAB-ZFP/ KAP1 complex induces a repressive chromatin state in the KRAB-ZFP binding regions. For example, among several hundred mouse KRAB-ZFPs, ZFP809 binds an endogenous copy of Mouse Leukemia Virus to induce DNA and histone H3K9 methylation in the internal sequence and LTRs [29]. Likewise, the human KRABZFPs, ZNF91 and ZNF93, bind to SVA and L1 retrotransposons, respectively, leading to an induction of histone H3K9 methylation [30]. It is possible that a certain KRAB-ZFP binds to the IAP internal sequence to induce DNA methylation. ZFP819 may be a candidate because the protein has been shown to bind the IAP internal sequence (5' UTR and the pol region of IAPEZ-int) and LTR (IAPLTR1a), and to regulate IAP expression [31]. In addition, not mutually exclusive, it is also possible that the internal sequence inhibits binding of the TET1, TET2, and/or TET3 enzymes, which catalyze oxidation of methylcytosine leading to loss of methylation [32].
It has been proposed that some genomic copies of retrotransposons can serve as a source of epigenetic and phenotypic diversity within a population [33]. IAP is of particular interest in this regard because several IAP copies have been shown to behave as metastable epialleles $[7,8]$. These copies in the $A^{v y}, A^{i a p}$, and $A x i n^{f u}$ alleles belong to the IAPLTR1 subfamily and not present in our mouse strain (C57BL6/J) [7, 8]. Thus, the mechanism for their occasional hypomethylation remains unknown. To understand the retrotransposon-mediated heritable epigenetic changes more profoundly, genomewide analyses of epigenetic states of these sequences must be performed for different tissues of many individuals with the discrimination of individual copies. The results here demonstrate that TEPBAT offers a costeffective method for such studies.

\section{Conclusions}

Using TEPBAT, we revealed that solo LTRs of specific subfamilies (IAPLTR2a2 and IAPEY in sperm and IAPLTR2 in tail) tend to be hypomethylated. Binding of TFs seems to account for hypomethylation of these copies, emphasizing the importance of TFs in regulating the epigenome. It should be noted, however, that the hypomethylation of IAPLTR2a2 and IAPEY copies in the 


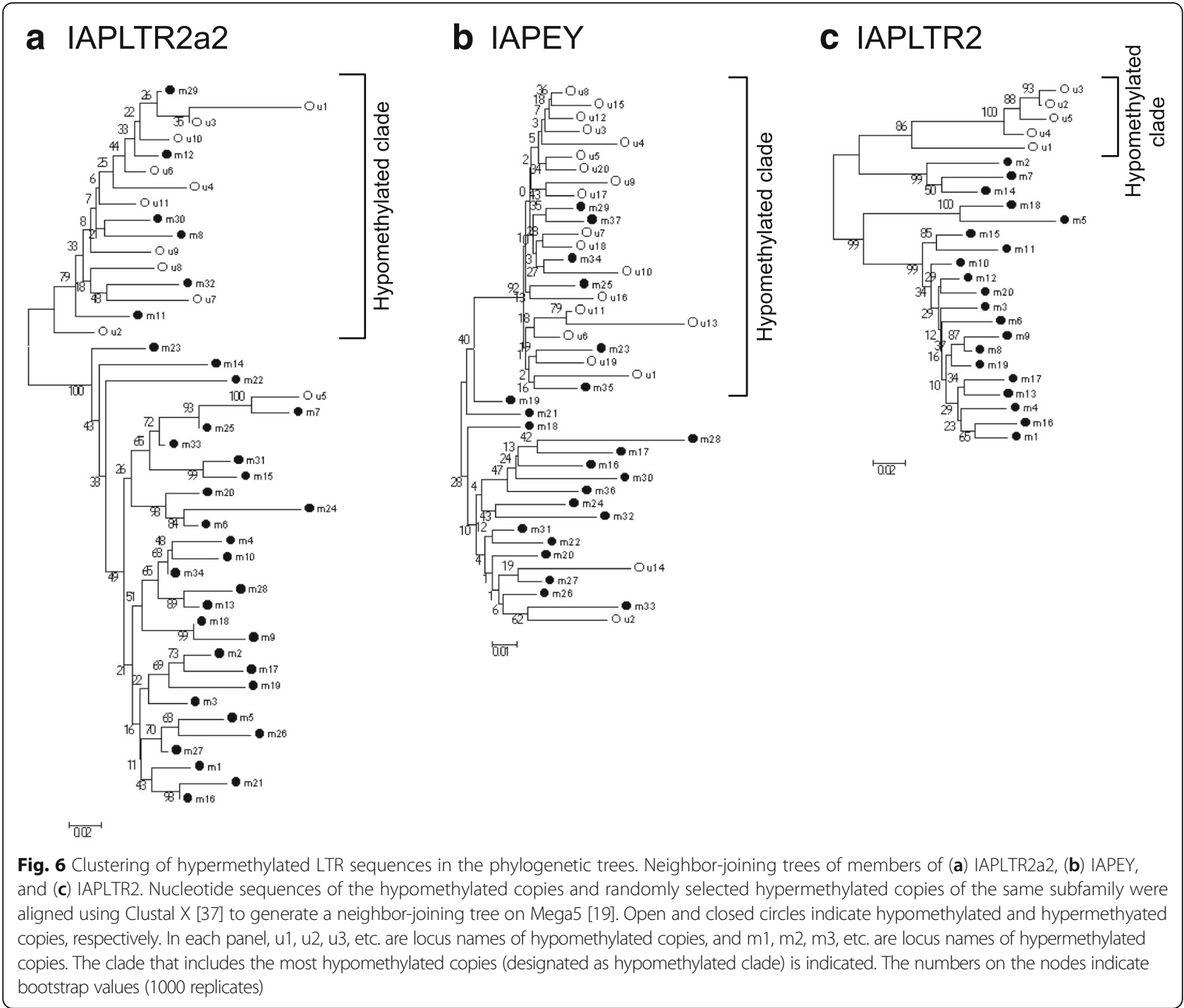

sperm does not explain the higher expression of IAP in spermatogenic cells because most of the hypomethylated copies were solo LTRs lacking the internal sequence. Although further studies will be required to solve the issue, our method can be applied to a variety of DNA methylome studies that focus on retrotransposon sequences.

\section{Methods}

Sequence library construction and paired-end sequencing High-molecular-weight genomic DNA was prepared from tail and sperm samples of the same adult mouse (C57BL6/ J) by a standard procedure. The DNA was treated with $10 \mathrm{M}$ bisulfite (sodium and ammonium salt), as described previously [34]. The resultant DNA (25 ng) was incubated with $80 \mathrm{nM}$ tag-plus-random primer (5'-GCAGTGAACTGACTACAGGNNNN-3') in Klenow buffer (10 mM TrisHCl, $10 \mathrm{mM} \mathrm{MgCl}_{2}, 1 \mathrm{mM}$ DTT, and $125 \mu \mathrm{M}$ each of dNTPs) at $94{ }^{\circ} \mathrm{C}$ for $5 \mathrm{~min}$, then quickly cooled down to
$4{ }^{\circ} \mathrm{C}$. After 7.5 U of Klenow Fragment exo ${ }^{-}$(New England Biolab) was added, the reaction mixture was incubated at $4{ }^{\circ} \mathrm{C}$ for $15 \mathrm{~min}$. The temperature was then increased to $37^{\circ} \mathrm{C}$ at the rate of $1{ }^{\circ} \mathrm{C}$ per minute and maintained at $37{ }^{\circ} \mathrm{C}$ for $90 \mathrm{~min}$. After heat inactivation at $70{ }^{\circ} \mathrm{C}$ for 10 min, DNA was purified using AMPure XP (Beckman Coulter). IAP-containing genomic regions were amplified by PCR $\left(25\right.$ cycles of $94{ }^{\circ} \mathrm{C}$ for $15 \mathrm{~s}, 55^{\circ} \mathrm{C}$ for $30 \mathrm{~s}$, and $68{ }^{\circ} \mathrm{C}$ for $30 \mathrm{~s}$ ) using KOD-plus neo (Toyobo), tag primer (5'-CAGTGAAC- TGACTACAGG-3'), and either of IAPBS1 (5'-GGGGAAGGTAGAGTATAWG-3') or IAP-BS2 (5'-GGTTTTTGAAGATGTAAGTAATAAAGTTTT-3') primers. The 350-450-bp long PCR products were purified by electrophoresis using a $2 \%$ agarose gel, and Illumina sequencing adaptors were added to their ends using the TruSeq DNA Sample Prep kit (Illumina). Paired-end 100-bp sequencing was carried out on a HiSeq2500 in the highoutput mode (one lane per tissue sample with equal 

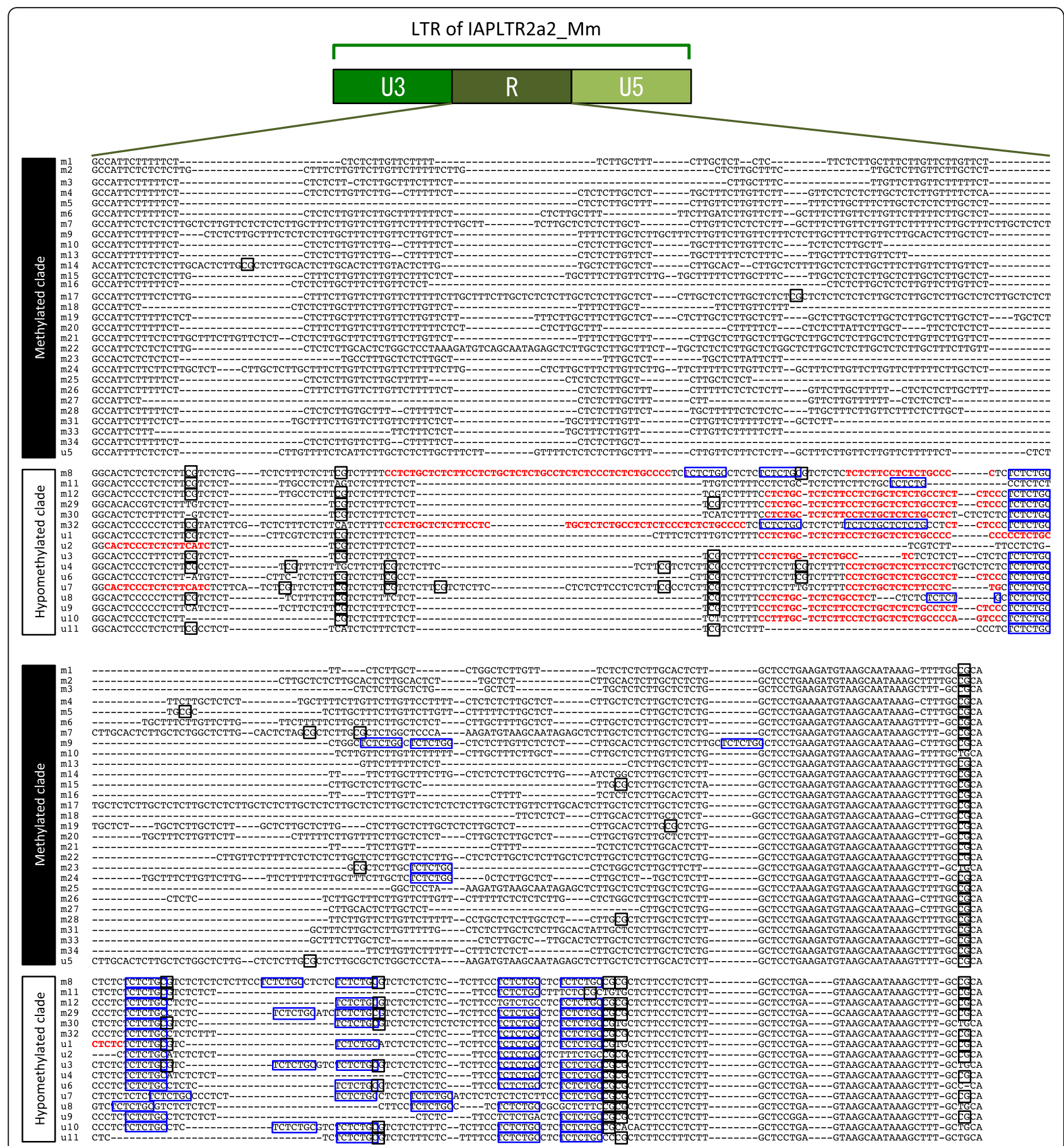

RED LETTERS: Maz binding motifs

BLUE BOX Ubp1 binding motifs

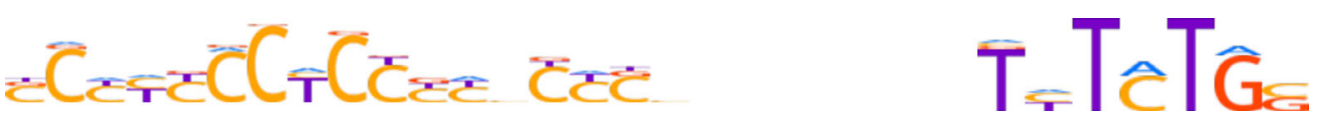

Fig. 7 Hypomethylated clade of IAPLTR2a2 is characterized by the presence of Maz- and Ubp1-binding motifs. The nucleotide sequences of the R region of the LTR (see top for a schematic view of the LTR sub-regions) of IAPLTR2a2 copies belonging to the methylated and hypomethylated clades were aligned by Clustal X [37]. Maz-binding motifs are highlighted in red. Ubp1-binding sites are indicated by a blue box. CpG sites are indicated by a black box. Members of the hypomethylated clade carry multiple Maz and Ubp1 sites, whereas almost all members of the hypermethylated clade carry none. Sequence Logo representation of the binding motifs is shown at the bottom 


\section{a full-length element

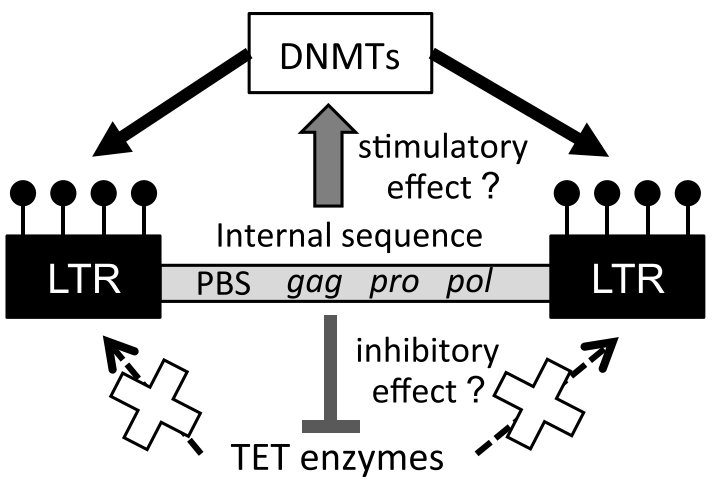

b
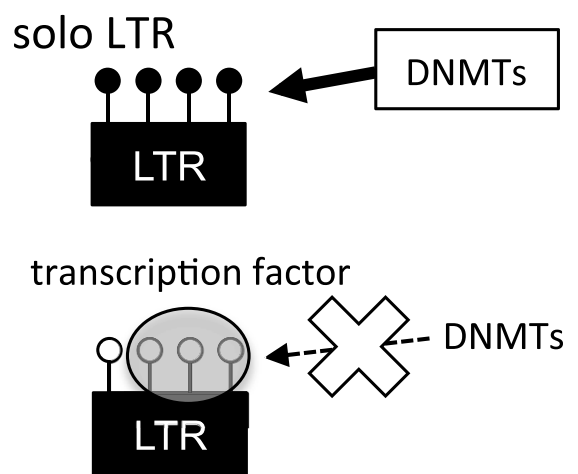

Fig. 8 Models for molecular mechanisms that regulate IAP methylation. a In copies containing the internal sequence, some parts of the internal sequence may stimulate DNA methylation in their flanking LTR sequences. Closed circles represent methylated CpG sites in LTRs. DNMTs, DNA methyltransferases. TET enzymes, Ten-Eleven translocation enzymes (catalyzing oxidation of methylcytosine). PBS, primer binding site. b Most solo LTR copies are methylated by DNMTs (top). However, copies carrying TFBSs bind to these TFs if the TFs are expressed in the cell. This binding inhibits the action of DNMTs (bottom). The bound transcription factor is represented as a gray oval. Open circles represent unmethylated $\mathrm{CpG}$ sites in the LTRs

amounts of the IAP-BS1 and IAP-BS2 libraries being mixed). About $80 \%$ of read pairs contained the tag and IAP-BS1/BS2 sequences. After removing the primer sequences (including the random tetramer region) and lowquality bases by a perl script, the read pairs were mapped to the genome (mm10) by Bismark [35] with default parameters to call the methylation state at each $\mathrm{CpG}$ position. With this set of parameters, read pairs with multiple hits were discarded. The mapping efficiency was $22-25 \%$. The low mapping efficiency in comparison to PBAT (typically $50-70 \%$ ) is likely due to the fact that many LTR copies are flanked by repeat elements such as IAP internal sequences.

\section{Bisulfite-PCR analysis}

Using the bisulfite-treated tail DNA, touch-down PCR was performed as described previously [34] with locus- specific primers (forward and reverse primers were designed for the LTR and flanking region, respectively). PCR fragments were cloned into the pGEM-Teasy vector (Promega) and sequenced using 3730 DNA Analyzer (Applied Biosystems).

\section{Methylation analysis of IAP LTR sequences}

The genomic positions of IAP sequences were obtained from the repeatmasker table from the UCSC genome browser [36]. For each LTR copy, their features (5' LTR, 3' LTR, or solo LTR) were manually determined (Table S1). To calculate LTR methylation levels, the methylation levels of CpG sites within the LTRs were averaged. Only those LTRs having $\geqq 20$ CpG methylation calls (sum of sequencing depth at CpG sites) were used for further analysis. Only LTRs present in the reference sequence were analyzed.

\section{Additional files}

Additional file 1: Sequence divergence of IAPEY_LTR genomic copies. (PDF 106 kb)

Additional file 2: Table S1. Annotation and methylation levels of each IAP fragment. (XLSX $2426 \mathrm{~kb}$ )

\section{Abbreviations}

H3K9: The lysine-9 residue of histone H3; IAP: Intracisternal A particle; KRABZFP: Krüppel-associated box containing zinc finger protein; LTR: Long terminal repeat; PCR: Polymerase chain reaction; SNV: Single nucleotide variation; TEPBAT: Target Enrichment after Post-Bisulfite Adaptor Tagging; TF: Transcription factor

\section{Acknowledgements}

We thank Dr. Hidehiro Toh and Ms. Miho Miyake for assistance in DNA sequencing.

\section{Funding}

Research grants from the Ministry of Education, Culture, Sports, Science, and Technology of Japan to KI (grant numbers 25,503,003, 16H04817, and 16 K14784) and to HS (JP25112010).

Research grant from the Takeda Science Foundation to KI.

\section{Availability of data and materials}

The dataset generated and analyzed during the current study is available at the Gene Expression Omnibus (GEO) under the accession number, GSE102989.

\section{Authors' contributions}

KS performed experiments and data analysis. KF performed experiments. HS supervised the study. KI designed the study, performed sequence analysis, and wrote the manuscript. All authors read and approved the final manuscript.

\section{Ethics approval}

The animal experiments were conducted according to Japanese Act on Welfare and Management of Animals, Guidelines for Proper Conduct of Animal Experiments (published by Science Council of Japan), Fundamental Guidelines for Proper Conduct of Animal Experiment and Related Activities in Academic Research Institutions (published by Ministry of Education, Culture, Sports, Science and Technology, Japan), and Regulation for Animal Experiments at Kyushu University and at Nagoya University. The protocols have been approved by Animal Experiments Committees in Kyushu University (A26-010-3) and in Nagoya University (2017042501).

Consent for publication

Not applicable. 


\section{Competing interests}

The authors declare that they have no competing interests.

\section{Publisher's Note}

Springer Nature remains neutral with regard to jurisdictional claims in published maps and institutional affiliations.

\section{Author details}

${ }^{1}$ Division of Epigenomics and Development, Medical Institute of Bioregulation, and Epigenome Network Research Center, Kyushu University, 3-1-1 Maidashi, Higashi-ku, Fukuoka 812-8582, Japan. ${ }^{2}$ Trygroup Incorporated, 1-8-10 Kudankita, Chiyoda-ku, Tokyo 102-0073, Japan. ${ }^{3}$ Cellular Memory Laboratory, RIKEN, Wako, Saitama 351-0198, Japan. ${ }^{4}$ Laboratory of Genome and Epigenome Dynamics, Department of Applied Molecular Biosciences, Graduate School of Bioagricultural Sciences, Nagoya University, Nagoya 464-8601, Japan.

Received: 7 September 2017 Accepted: 1 December 2017

Published online: 13 December 2017

\section{References}

1. Mouse Genome Sequencing, C, Waterston RH, Lindblad-Toh K, Birney E, Rogers J, Abril JF, Agarwal P, Agarwala R, Ainscough R, Alexandersson M, et al. Initial sequencing and comparative analysis of the mouse genome. Nature. 2002;420:520-62.

2. Belancio VP, Hedges DJ, Deininger P. Mammalian non-LTR retrotransposons: for better or worse, in sickness and in health. Genome Res. 2008;18:343-58.

3. Maksakova IA, Romanish MT, Gagnier L, Dunn CA, van de Lagemaat LN, Mager DL. Retroviral elements and their hosts: insertional mutagenesis in the mouse germ line. PLoS Genet. 2006;2:e2

4. Walsh CP, Chaillet JR, Bestor TH. Transcription of IAP endogenous retroviruses is constrained by cytosine methylation. Nat Genet. 1998;20:116-7.

5. Karimi MM, Goyal P, Maksakova IA, Bilenky M, Leung D, Tang JX, Shinkai $Y$, Mager DL, Jones S, Hirst M, et al. DNA methylation and SETDB1/H3K9me3 regulate predominantly distinct sets of genes, retroelements, and chimeric transcripts in mESCs. Cell Stem Cell. 2011;8:676-87.

6. Matsui $T$, Leung D, Miyashita $H$, Maksakova IA, Miyachi $H$, Kimura $H$ Tachibana M, Lorincz MC, Shinkai Y. Proviral silencing in embryonic stem cells requires the histone methyltransferase ESET. Nature. 2010;464:927-31.

7. Morgan HD, Sutherland HG, Martin DI, Whitelaw E. Epigenetic inheritance at the agouti locus in the mouse. Nat Genet. 1999;23:314-8.

8. Rakyan VK, Chong S, Champ ME, Cuthbert PC, Morgan HD, Luu KV, Whitelaw E. Transgenerational inheritance of epigenetic states at the murine Axin(fu) allele occurs after maternal and paternal transmission. Proc Natl Acad Sci U S A. 2003;100:2538-43.

9. Svoboda P, Stein P, Anger M, Bernstein E, Hannon GJ, Schultz RM. RNAi and expression of retrotransposons MuERV-L and IAP in preimplantation mouse embryos. Dev Biol. 2004;269:276-85.

10. Dupressoir A, Heidmann T. Germ line-specific expression of intracisternal Aparticle retrotransposons in transgenic mice. Mol Cell Biol. 1996;16:4495-503.

11. Lane N, Dean W, Erhardt S, Hajkova P, Surani A, Walter J, Reik W. Resistance of IAPs to methylation reprogramming may provide a mechanism for epigenetic inheritance in the mouse. Genesis. 2003;35:88-93.

12. Reik W, Dean W, Walter J. Epigenetic reprogramming in mammalian development. Science. 2001;293:1089-93.

13. Sasaki $H$, Matsui $Y$. Epigenetic events in mammalian germ-cell development: reprogramming and beyond. Nat Rev Genet. 2008;9:129-40.

14. Ekram MB, Kim J. High-throughput targeted repeat element bisulfite sequencing (HT-TREBS): genome-wide DNA methylation analysis of IAP LTR retrotransposon. PLoS One. 2014;9:e101683.

15. Miura F, Enomoto Y, Dairiki R, Ito T. Amplification-free whole-genome bisulfite sequencing by post-bisulfite adaptor tagging. Nucleic Acids Res. 2012:40:e136.

16. Fennelly J, Harper K, Laval S, Wright E, Plumb M. Co-amplification to tail-totail copies of MuRVY and IAPE retroviral genomes on the Mus Musculus $Y$ chromosome. Mamm Genome. 1996;7:31-6.

17. Hammoud SS, Low DH, Yi C, Carrell DT, Guccione E, Cairns BR. Chromatin and transcription transitions of mammalian adult germline stem cells and spermatogenesis. Cell Stem Cell. 2014;15:239-53.

18. Kobayashi H, Sakurai T, Miura F, Imai M, Mochiduki K, Yanagisawa E, Sakashita A, Wakai T, Suzuki Y, Ito T, et al. High-resolution DNA methylome analysis of primordial germ cells identifies gender-specific reprogramming in mice. Genome Res. 2013:23:616-27.

19. Tamura K, Peterson D, Peterson N, Stecher G, Nei M, Kumar S. MEGA5 molecular evolutionary genetics analysis using maximum likelihood, evolutionary distance, and maximum parsimony methods. Mol Biol Evol. 2011;28:2731-9.

20. Grant CE, Bailey TL, Noble WS. FIMO: scanning for occurrences of a given motif. Bioinformatics. 2011:27:1017-8.

21. Christy RJ, Brown AR, Gourlie BB, Huang RC. Nucleotide sequences of murine intracisternal A-particle gene LTRs have extensive variability within the $R$ region. Nucleic Acids Res. 1985;13:289-302.

22. Inoue K, Ichiyanagi K, Fukuda K, Glinka M, Sasaki H. Switching of dominant retrotransposon silencing strategies from posttranscriptional to transcriptional mechanisms during male germ-cell development in mice. PLoS Genet. 2017:13:e1006926.

23. Banovich NE, Lan X, McVicker G, van de Geijn B, Degner JF, Blischak JD, Roux J, Pritchard JK, Gilad Y, Methylation QTL. Are associated with coordinated changes in transcription factor binding, histone modifications, and gene expression levels. PLoS Genet. 2014;10:e1004663.

24. Fukuda K, Ichiyanagi K, Yamada Y, Go Y, Udono T, Wada S, Maeda T, Soejima $H$, Saitou N, Ito T, et al. Regional DNA methylation differences between humans and chimpanzees are associated with genetic changes, transcriptional divergence and disease genes. J Hum Genet. 2013;58:446-54

25. Fukuda K, Inoguchi Y, Ichiyanagi K, Ichiyanagi T, Go Y, Nagano M, Yanagawa Y, Takaesu N, Ohkawa Y, Imai H, et al. Evolution of the sperm methylome of primates is associated with retrotransposon insertions and genome instability. Hum Mol Genet. 2017;26:3508-19.

26. Gutierrez-Arcelus M, Lappalainen T, Montgomery SB, Buil A, Ongen H, Yurovsky A, Bryois J, Giger T, Romano L, Planchon A, et al. Passive and active DNA methylation and the interplay with genetic variation in gene regulation. elife. 2013;2:e00523.

27. Lupo A, Cesaro E, Montano G, Zurlo D, Izzo P, Costanzo P. KRAB-zinc finger proteins: a repressor family displaying multiple biological functions. Curr Genomics. 2013;14:268-78.

28. Urrutia R. KRAB-containing zinc-finger repressor proteins. Genome Biol. 2003;4:231.

29. Wolf D, Goff SP. Embryonic stem cells use ZFP809 to silence retroviral DNAs. Nature. 2009;458:1201-4

30. Jacobs FM, Greenberg D, Nguyen N, Haeussler M, Ewing AD, Katzman S, Paten B, Salama SR, Haussler D. An evolutionary arms race between KRAB zinc-finger genes ZNF91/93 and SVA/L1 retrotransposons. Nature. 2014;516:242-5.

31. Tan X, Xu X, Elkenani M, Smorag L, Zechner U, Nolte J, Engel W, Pantakani DV. Zfp819, a novel KRAB-zinc finger protein, interacts with KAP1 and functions in genomic integrity maintenance of mouse embryonic stem cells. Stem Cell Res. 2013;11:1045-59.

32. Wu X, Zhang Y. TET-mediated active DNA demethylation: mechanism, function and beyond. Nat Rev Genet. 2017;18:517-34.

33. Whitelaw E, Martin DI. Retrotransposons as epigenetic mediators of phenotypic variation in mammals. Nat Genet. 2001;27:361-5.

34. Ichiyanagi K, Li Y, Watanabe T, Ichiyanagi T, Fukuda K, Kitayama J, Yamamoto Y, Kuramochi-Miyagawa S, Nakano T, Yabuta Y, et al. Locus- and domain-dependent control of DNA methylation at mouse B1 retrotransposons during male germ cell development. Genome Res. 2011; 21:2058-66.

35. Krueger F, Andrews SR. Bismark: a flexible aligner and methylation caller for Bisulfite-Seq applications. Bioinformatics. 2011;27:1571-2.

36. Tyner C, Barber GP, Casper J, Clawson H, Diekhans M, Eisenhart C, Fischer CM, Gibson D, Gonzalez JN, Guruvadoo L, et al. The UCSC genome browser database: 2017 update. Nucleic Acids Res. 2017;45:D626-34.

37. Thompson, J. D., Gibson, T. J. and Higgins, D. G. Multiple sequence alignment using ClustalW and ClustalX. Curr Protoc Bioinformatics. 2002: Chapter 2:Unit 23. 